\title{
IDENTIFICATION AQUIFER PARAMETERS THROUGH SINGLE WELL PUMPING TEST SERIES AT PT. KALTIM KARIANGAU TERMINAL, BALIKPAPAN, EAST KALIMANTAN
}

\author{
Totok Sulistyo ${ }^{1 *}$ \\ ${ }^{1}$ Civil Engineering Dept, Balikpapan State Polytechnics \\ JI. Soekarno Hatta, Km.8 Balikpapan \\ E-mail: totok.sulistyo@poltekba.ac.id
}

\begin{abstract}
Aquifer Parameters are very important in groundwater and well management. The objective of this research is to determine aquifer parameter in order to be used in determining suitable production rate of well. Research was carried out in PT. Kaltim Kariangau Terminal, which is administratively, located in Balikpapan City, East Kalimantan, Indonesia. PT. Kaltim Kariangau Terminal has developed four wells with distance of each of well is between 50 and 300 meters, but it is a pity because just one well was completed by pumping test without observation well. Result of constant pumping test analyzing through Cooper - Jacob method has shown that value of Transmissivity (T) of aquifer is $319.0718283 \mathrm{~m} 2 /$ day, and it is known from geophysical logging and well construction design that the thickness of aquifer is $48 \mathrm{~m}$, so hydraulic conductivity (K) of aquifer is $6.6473 \mathrm{~m} / \mathrm{day}$. Coefficient of aquifer loss is 0.0013 and coefficient of well loss is 0.0000008 . Factors development of well could be classified as very effective with the well condition is properly designed and developed.
\end{abstract}

Keyword: groundwater, aquifer, transmissivity, hydraulic conductivity.

\section{INTRODUCTION}

Human body consists of almost $50-70 \%$ of water (Slamet, 1994), therefore it is undeniable that water is vital source of life, even bad quality of water in Indonesia has been reported for causing up to $88 \%$ dead of child (UNICEF, 2012). It has been known for thousand years ago, in order to support human living, people has been using many source of water such as, rain, stream, and groundwater. In many area that geologically have ground water reserves most people get ground water through, spring, qanats, artesian well, dug well and drilled deep well.

Groundwater reserves in this world is $0.61 \%$ and surface water is $0.009 \%$ from total groundwater reserves (Fetter, 1994). This means ratio between surface water and groundwater is $1 / 67.78$. Now day, where the existing of ground water could be detected through many tools such as hydrogeological knowledge, geophysical survey so that, groundwater have been explored and exploited much more.

Groundwater exploitation through deep well has become alternative to get more water which is needed in abundant amount for various industry need, such as hotels, textile, drinking water, hospital, agriculture etc. Recently, the advance of drilling technology and subsurface knowledge enable well getting much deeper that cross cut several aquifers systems so it could collect more water and increase well productivity. Meanwhile well productivity will be determined by aquifer parameters such as hydraulic conductivity, transmissivity, and well construction design or development factors of well. So that getting data of Transmissivity and hydraulic conductivity is paramount, because transmissivity and hydraulic conductivity are among the most important hydrogeological data needed for managing groundwater resources (Kumar et al., 2014).

The installation of water pump is done after serial of pumping test conducted. The series of pumping tests consist of constant rate test/long term test, recovery test, steps drawdown test. The one final aim of those test is to determine the feasibility of well, optimum pumping rate, and suitable pump specification.

The research objective is to determine parameters of aquifer such as hydraulic conductivity and transmissibility. This research is also aimed to determine well loss, aquifer loss, development factors of well.

Research was carried out in PT. Kaltim Kariangau Terminal. Administratively, Research area is located in Balikpapan City, East Kalimantan, Indonesia. According to the planning of Development Planning Body of Balikpapan City and East Kalimantan Province, 
it will be developed as seaport with its facilities and integrated with industrial area to support the development of Kariangau Special Economic Zone.

\section{RESEARCH METHOD}

This research involved many activities, starting from desk study, collecting data, analyzing and drawing conclusion. Technical data such as geophysical logging, well construction design and pumping test data were obtained from management of PT. Kaltim Kariangau Terminal as owner of the project. Other secondary data was collecting from Balikpapan City Development Planning Body such geological and city spatial planning map.

In order to have better understanding of the underlying aquifer, Cooper-Jacob method was employed to analyze pumping test data of the well and to get aquifers parameter such as transmissivity, hydraulic conductivity, aquifer loss, well loss, development factor and efficiency of well. Geological interpretation was done to identify potential environmental impacts the activity of ground water pumping in long term.

PT. Kaltim Kariangau Terminal has developed four wells, and as measured by GPS their location in UTM respectively; Well KKT DWW \# 01 Easting 0476364 m, Northing $9872219 \mathrm{~m}$, well KKT DWW \#02 Easting $0476315 \mathrm{~m}$, Northing $9872161 \mathrm{~m}$ well KKT DWW \#03 Easting $0476191 \mathrm{~m}$, Northing 9872169 m KKT DWW \#04 Easting 0476291 m, Northing $9871811 \mathrm{~m}$.

It is a pity from four wells only one well was completed with pumpping test without observation well. These well is located in Kutai Basin, they have depth more than 200 meters. Geologically, it passes through Pleistocene Kampung Baru Formation which is dominated by silt and clay. The underneath formation is member of Balikpapan Group (Late Miocene Sepinggan Formation) which is characterized by sandstone slit clay and coal (Satyana et al., 1999) as showing by geological map and geophysical logging profile. Having three other close wells have no pumping test data, they can't be further more analyzed for both of their hydraulic conductivity and transmissivity

\section{RESULT AND DISCUSSION}

According to Cooper - Jacob Method in Todd, 1980 a value of transmissivity $(T)$ is governed by this formula:

$T=\frac{2.30 Q}{4 \pi \Delta s}$
From the first equation, it can be simplified become equation 2 :

$T=1,183 Q / \Delta S^{\prime}$

Where $\mathrm{Q}$ is pumping rate, $\Delta s$ is drawdown difference per log cycle

And hydraulic conductivity can be solved as follow:

$\mathrm{K}=\mathrm{T} / \mathrm{b}$

Where $b$ is aquifer thickness.

And specific capacity (Sc) can be calculated using the following formula

$\mathrm{Sc}=\mathrm{Q} / \mathrm{SW}$

Here is the determination of transmissivity and hydraulic conductivity through analysis of continuous pumping, recovery and step drawdown test.

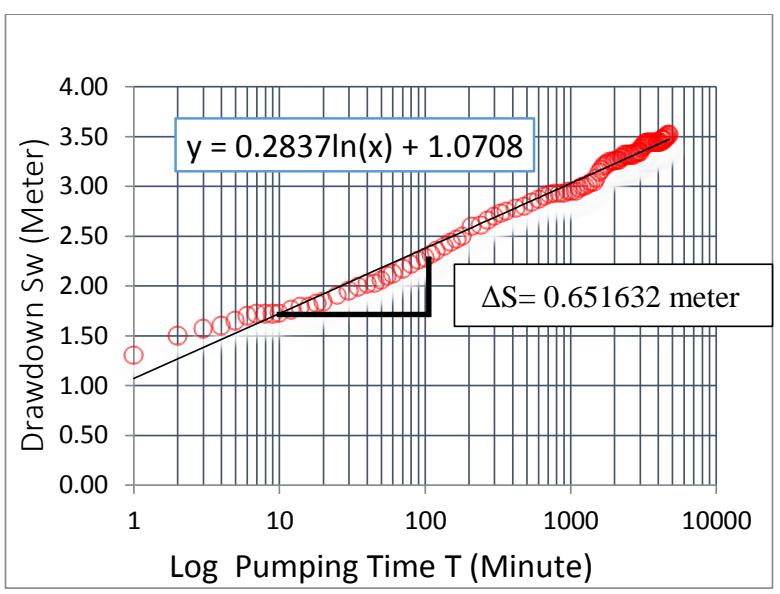

Figure 1. Semilogarithmic plot of constant pumping rate test

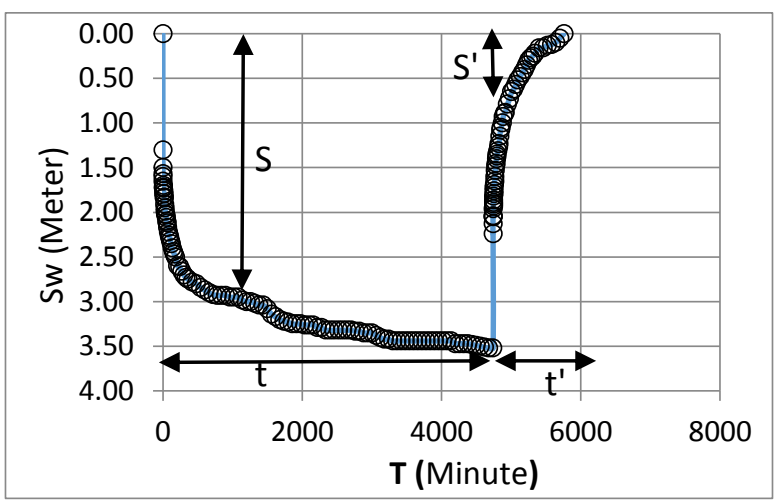

Figure 2. Constant pumping rate and recovery plot,

From constant pumping rate plot in Figure 1, it can be calculated transmissivity ( $\mathrm{T}$ ) and 
hydraulic conductivity $\mathrm{K}$ of aquifer as follow,

Equilibrium of the curve is:

$y=0.283 \ln (x)+1.070$

Then drawdown per log cycle can be determined as follow;

$\Delta S=y 2-y 1=y(100)-y(10)$

$\Delta S=0.283 \ln (100)+1.070-0.283 \ln (10)+1.070$

$\Delta S=2,373263163-1,721631581$

$\Delta S=0.651632$ meter

And transmissivity

$T=1,183 Q / \Delta S$

$T=1,183 * 0,01315 / 0,651632$

$T=0,003692961 \mathrm{~m}^{2} /$ second

$T=319.0718283 \mathrm{~m}^{2} /$ day

the thickness of aquifer (b) is 48 meters (known from geophysical logging profile and length of screen in well construction design) then hydraulic conductivity can be calculated using this equation 3 :

$$
\begin{aligned}
& K=T / b \\
& K=319.0718283 / 48 \\
& K=6.6473 \mathrm{~m} / \text { day }
\end{aligned}
$$

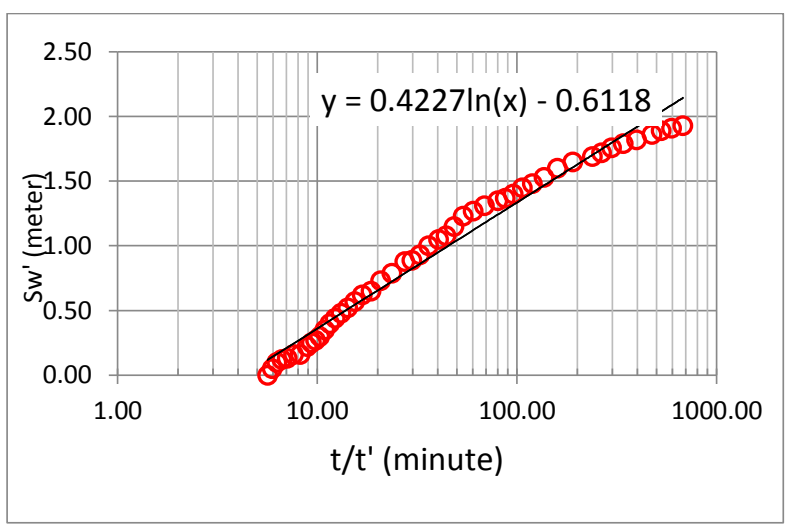

Figure 3. Semilogarithmic plot of constant pumping recovery

We can compare the above $\mathrm{T}$ and $\mathrm{K}$ parameters from its recovery as calculated bellow (see Figure 3).

$y=0.422 \ln (x)-0.611$
$\Delta S^{\prime}=y 2-y 1=y(100)-y(10)$

$\Delta S^{\prime}=(0.422 \ln (100)-0.611)-(0.422 \ln (10)$ $-0.611)$

$\Delta S=1.333303-0.361151$

$\Delta S=0.972151$ meter

$T=1,183 Q / \Delta S^{\prime}$

$T=1.183 * 0,01315 / 0,972151$

$T=0.002475 \mathrm{~m}^{2} /$ second

$T=213.8733 \mathrm{~m}^{2} /$ day

$K=213.8733 / 48$

$K=4.4557 \mathrm{~m} /$ day

Using similar method of calculation of transmissivity $(T)$ and hydraulic conductivity $(K)$ as above calculation, here is the result of calculation of $\mathrm{T}$ and $\mathrm{K}$ from step drawdown test.

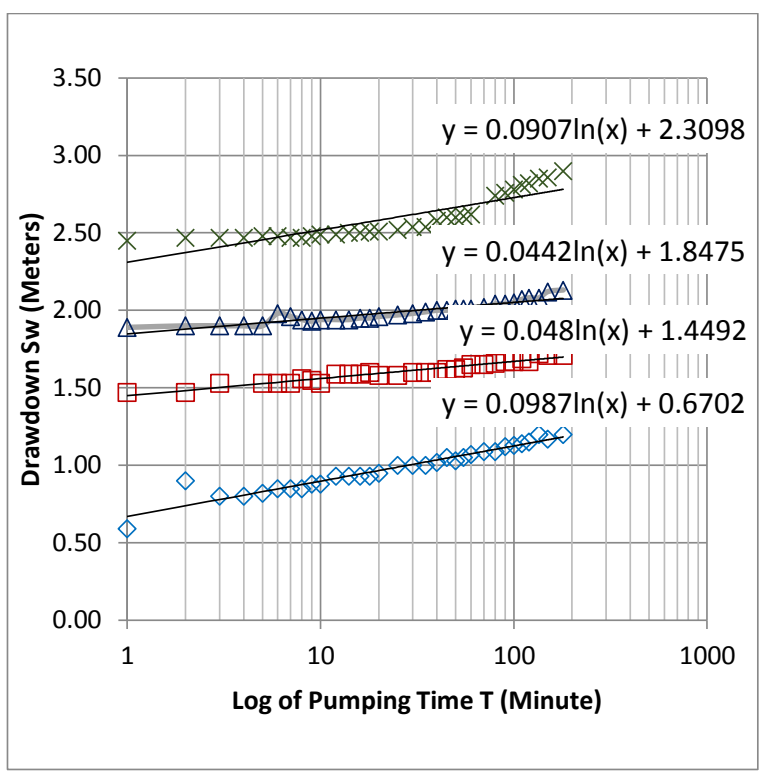

Figure 4. Semilogarithmic plot of step drawdown test 
Table 1. Pumping rate, Transmissivity and Hydraulic Conductivity of each step

\begin{tabular}{|c|c|c|c|c|}
\hline & $\begin{array}{l}\text { Pumping } \\
\text { Rate } \\
\text { (Q) } \\
\text { m³ day }^{3} \text { dat }\end{array}$ & $\begin{array}{c}\text { Transmis } \\
\text { sivity (T) } \\
\mathrm{m}^{2} / \text { day }\end{array}$ & $\begin{array}{c}\text { Hydraulic } \\
\text { Conductiv } \\
\text { ity K } \\
\text { m/day }\end{array}$ & $\begin{array}{c}\text { Specific } \\
\text { Capacity } \\
\text { (Sc) } \\
\mathrm{m}^{2} / \text { day }\end{array}$ \\
\hline $\begin{array}{c}\text { STEP } \\
\text { S }\end{array}$ & 624.6720 & 506.5955 & 10.54407 & 520.56 \\
\hline $\begin{array}{l}\text { STEP } \\
\text { II }\end{array}$ & 978.0480 & 1616.54 & 33.67791 & $\begin{array}{c}575.322 \\
3\end{array}$ \\
\hline $\begin{array}{l}\text { STEP } \\
\text { III }\end{array}$ & $\begin{array}{c}1046.304 \\
0\end{array}$ & 1888.347 & 39.3406 & $\begin{array}{c}491.222 \\
5\end{array}$ \\
\hline $\begin{array}{l}\text { STEP } \\
\text { IV }\end{array}$ & $\begin{array}{c}1136.160 \\
0\end{array}$ & 1007.118 & 20.9816 & $\begin{array}{c}391.779 \\
3\end{array}$ \\
\hline
\end{tabular}

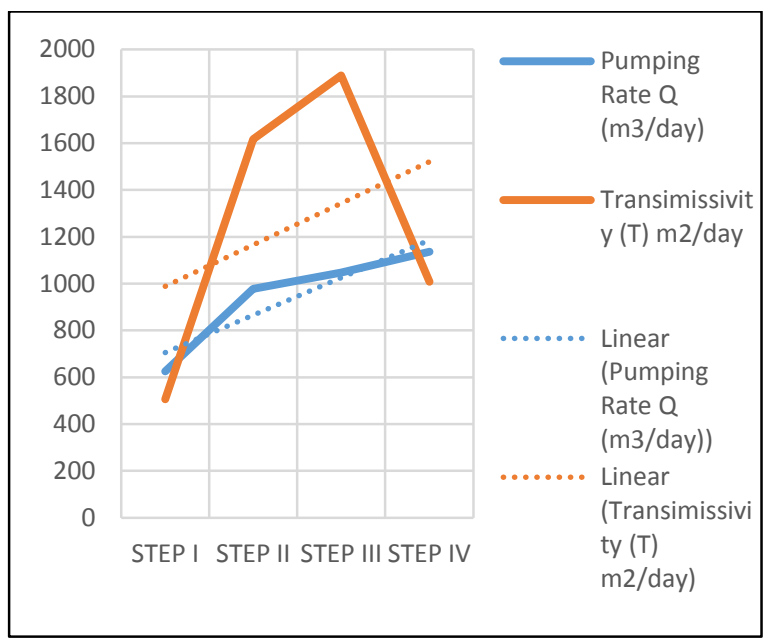

Figure 5. Correlation of pumping rate and transmissivity,

According to the classification of transmissivity magnitude (Krásný, 1993), The class transmissivity magnitude of aquifer in research area is II, high magnitude, groundwater supply potential is Withdrawals of lesser regional importance, with very approximate discharge of single well 5 up to 50 liters/second.

Transmissivity $(T)$ is defined as average of all horizontal hydraulic conductivities at various depths multiplied by the vertical saturated thickness of aquifer (Nielsen, 1991). Transmissivity has dimension square length per time that means velocity per wide unit. There is positive correlation between pumping rate and transmissivity as showing by trend line of chart that prove that velocity of groundwater toward well getting bigger as pumping rate increase.

Drawdown in the well consist of aquifer loss and well loss, that can be solved using step drawdown test data as follow:

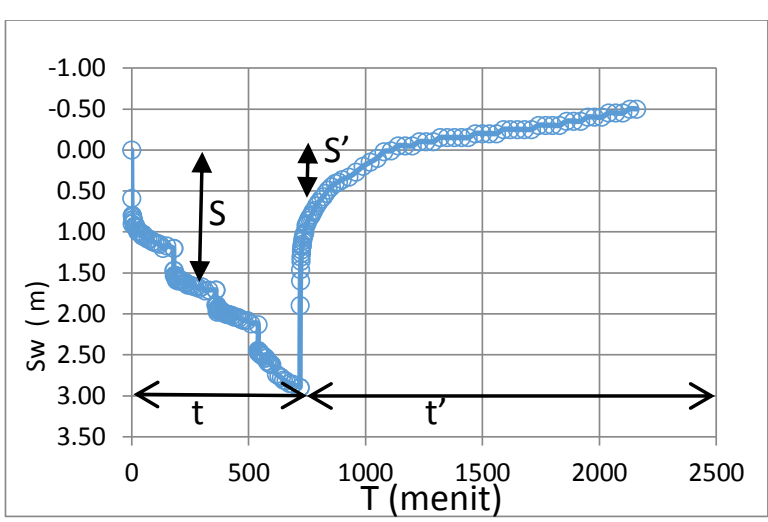

Figure 6. Step drawdown test and recovery plot.

The following chart gives illustration volume of water that has been discharge during step drawdown test and volume of water that has been naturally filled back during recovery test.

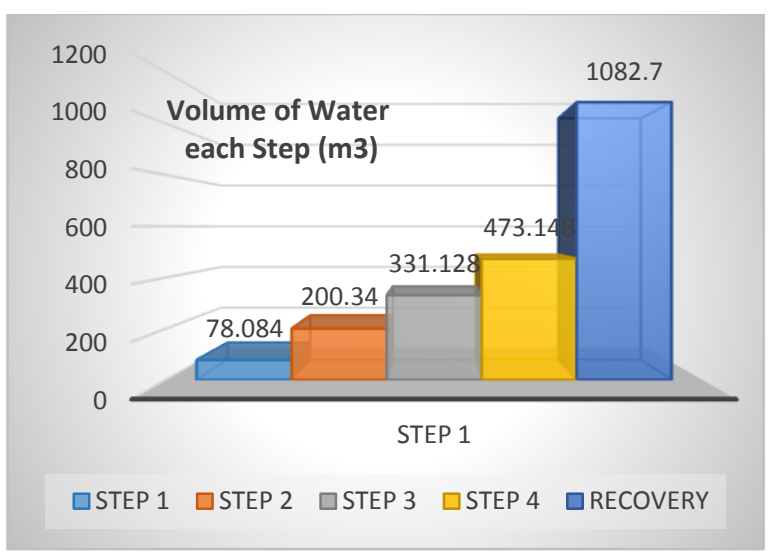

Figure 7. Volume of water in each step and recovery test.

Table 2. Pumping Rate, Drawdown, Drawdown/Pumping Rate

\begin{tabular}{lcccc}
\hline & $\begin{array}{c}\text { Pumping } \\
\text { Rate (Q) It/s }\end{array}$ & $\begin{array}{c}\text { Pumping Rate } \\
(\mathrm{Q}) \mathrm{m}^{3} / \text { day }\end{array}$ & $\begin{array}{c}\text { Draw } \\
\text { down } \\
(\mathrm{Sw}) \mathrm{m}\end{array}$ & Sw/Q \\
\hline STEP 1 & 7,23 & 624,6720 & 1,20 & 0.001921 \\
STEP 2 & 11,32 & 978,0480 & 1,71 & 0.001748 \\
STEP 3 & 12,11 & 1046,3040 & 2,13 & 0.002036 \\
STEP 4 & 13,15 & 1136,1600 & 2,90 & 0.002552 \\
\hline
\end{tabular}




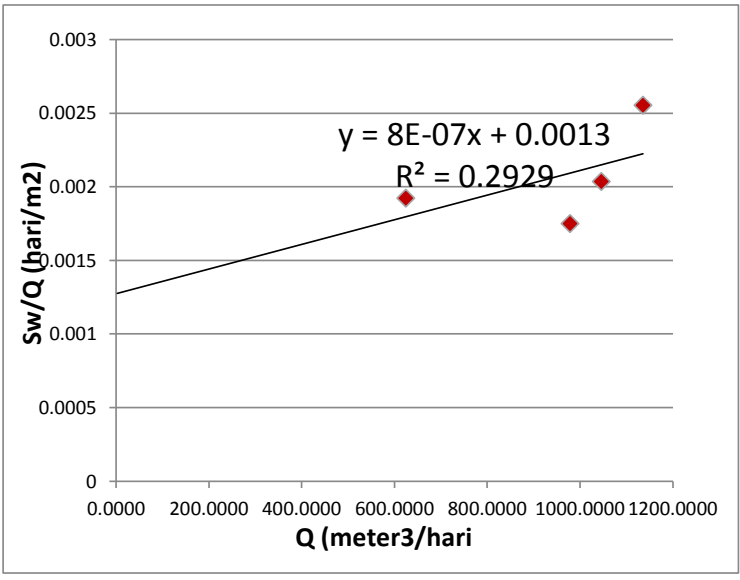

Figure 8. Linear plot to determine coefficient aquifer loss and well loss.

If $\mathrm{Sw}$ designs the drawdown inside the well in meter, $\mathrm{B}$ the formation resistance, $\mathrm{C}$ well loss and $Q$ the discharge $\left(\mathrm{m}^{3} \mathrm{day}^{-1}\right)$ we can write :

$\mathrm{Sw}=\mathrm{BQ}+\mathrm{CQ}^{2}$

(Chenini, 2008) furthermore in order to find linier of pumping test. The equation (4) can be made become equation (5)

$\mathrm{Sw} / \mathrm{Q}=\mathrm{B}+\mathrm{CQ}$.

From linier plot of figure $8, B$ and $C$ can be determined

$S w / Q=0.0013+0.0000008 Q$
$S w=0.0013 Q+0.0000008 Q^{2}$

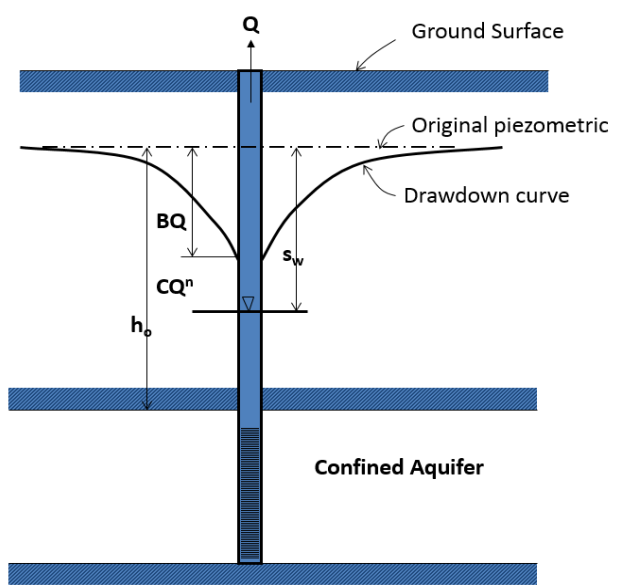

Figure 9. Aquifer Loss and Well Loss (Tod, 1980)

Factor development of well

$F d=\frac{C}{B} \cdot 100$

$$
=(0.0000008 / 0.0013) * 100
$$

$$
=0.0615
$$

Value of factor development (Fd) of well less than $0.1 \mathrm{day} / \mathrm{m}^{3}$ well can be classified as "very effective" (Bierschenk, 1963). Moreover value of $C$ less than 0.5 minute $2 / \mathrm{m}^{5}$ can be classified well condition is "properly designed and developed" (Walton, 1962 in Tod, 1980)

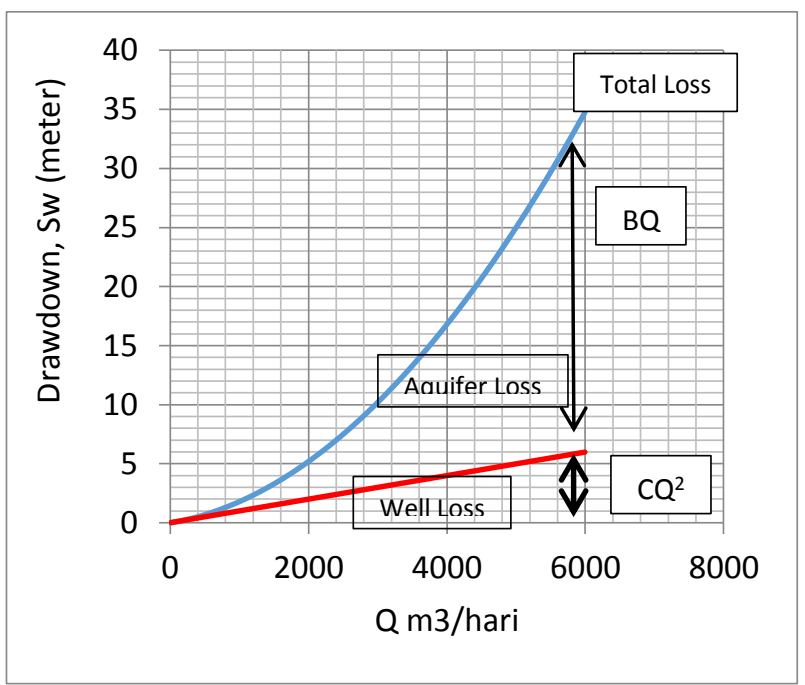

Figure 10. Simulated aquifer loss, well loss.

In the operation planning, pumping rate of KKT DWW \# 01 will be $0.001 \mathrm{~m}^{3} /$ second or $86.4 \mathrm{~m}^{3} /$ day, so that the well lost can be calculated as follow

$$
\begin{aligned}
\mathrm{CQ}^{2} & =0.0000008 * 86.4 * 86.4 \\
& =0.0059 \mathrm{~m} \\
& =5.9 \mathrm{~mm}
\end{aligned}
$$

And aquifer loss

$$
\begin{aligned}
\mathrm{BQ} & =0.0013^{\star} 86.4 \\
& =0.1123 \mathrm{~m} \\
& =112.3 \mathrm{~mm}
\end{aligned}
$$

Then total loss is

$$
\begin{aligned}
S w & =112.3+5.9 \\
& =118.2 \mathrm{~m}
\end{aligned}
$$

Through computer simulation well efficiency Ew, given as percentage (Tod, 1980) by

$E_{w}=100 \cdot \frac{B Q}{S_{w}}$

we can develop chart of well efficiency versus Pumping Rate as below. 


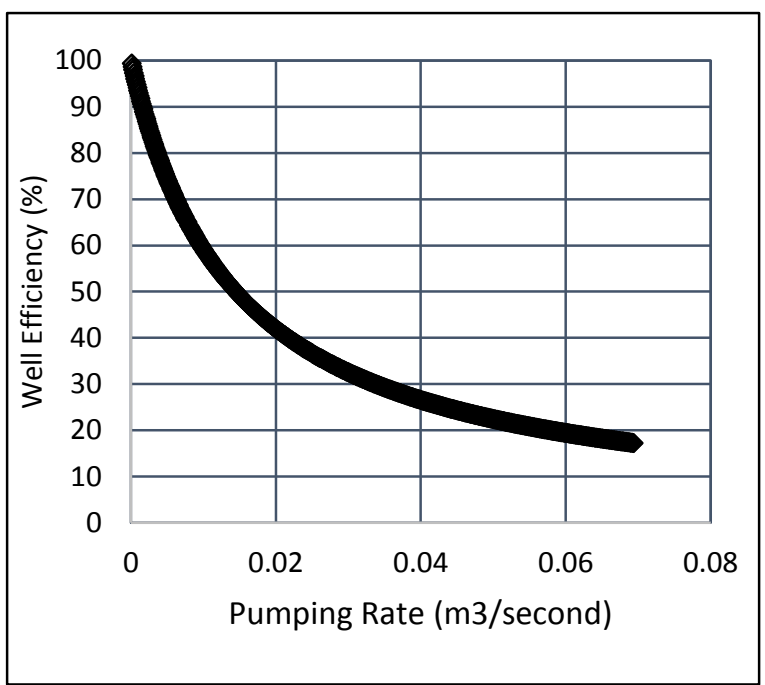

Figure 11. Simulated well efficiency plot.

In the pumping rate 10 liters/second or 0.001 $\mathrm{m}^{3} /$ second, KKT DWW \# 01 well efficiency can reach $93.53 \%$.

\section{CONCLUSION AND RECOMMENDATION}

According to KKT DWW \# 01 well data, after analyzing using Cooper-Jacob method it can be concluded that transmissivity from constant long term test is $319.0718283 \mathrm{~m}^{2} /$ day and hydraulic conductivity $6.6473 \mathrm{~m} / \mathrm{day}$. At the pumping rate 10 liters/second well efficiency is $93.53 \%$. Coefficient of aquifer loss is 0.0013 and coefficient of well loss is 0.0000008 . Factors development of well could be classified as very effective with the well condition is properly designed and developed. The transmissivity is high magnitude, groundwater supply potential is Withdrawals of lesser regional importance.

Although technically this well feasible for production with pumping rate 10 liters/second, but there three other well which is located close each other and their location close the coast therefore, so that it is highly recommended that wells should be monitored routinely in order to avoid salt water upcoming in the interface of freshwater and salt water, and other possible environmental impacts.

\section{ACKNOWLEDGMENTS}

Special thank goes to the management of PT. Kaltim Kariangau Terminal who has make this research possible by allowing author to access the available data. The author also would like to extend gratitude to the management of Poltekba who has encourage us to in order to be productive lecturer in research. Special thank also goes to my colleague Ms. Sara Wibaning Respati who has polished this manuscript grammatically so it more readable.

\section{REFERENCES}

Bierschenk, W. H. (1963). Determining well efficiency by multiple step-drawdown tests (pp. 493-507). International Association of Scientific Hydrology.

Chenini, I., Silvain, R., \& Ben-Mammou, A. (2008). A simple method to estimate Transmissibility and Storativity of Aquifer Using Specific Capacity of Wells. J. Applied Sci, 8(14), 2640-2643.

Fetter, C. W. (1994). Applied Hydrogeology Third Edition University of WisconsinOshkosh. Mc Millian College Publishing Company, New York.

Krásný, J. (1993). Classification of transmissivity magnitude and variation. Groundwater, 31(2), 230-236.

Kumar, T. J. R., Balasubramanian, A., Kumar, R. S., Dushiyanthan, C., Thiruneelakandan, B., Suresh, R., ... \& Davidraju, D. (2016). Assessment of groundwater potential based on aquifer properties of hard rock terrain in the Chittar-Uppodai watershed, Tamil Nadu, India. Applied Water Science, 6(2), 179186.

Nielsen, D. M. (Ed.). (1991). Practical handbook of ground-water monitoring. CRC Press.

Satyana, A. H., Nugroho, D., \& Surantoko, I. (1999). Tectonic controls on the hydrocarbon habitats of the Barito, Kutei, and Tarakan Basins, Eastern Kalimantan, Indonesia: major dissimilarities in adjoining basins. Journal of Asian Earth Sciences, 17(1-2), 99-122.

Slamet, J. S. (1994). Kesehatan lingkungan. Yogyakarta: Universitas Gadjah Mada.

Todd, D. K. (1980). Groundwater hydrology 2ed. John Wiley.

Unicef. (2012). Air bersih, sanitasi, dan kebersihan. Ringkasan Kajian UNICEF Indonesia. 
Appendix 1. Step Test Data of KKT DWW \# 01

Pumping Test Data

Step 1

\begin{tabular}{|c|c|c|c|c|c|c|}
\hline Date & Time & $\begin{array}{l}\text { Min } \\
\text { ute }\end{array}$ & $\begin{array}{l}\text { DWL } \\
\text { (m) }\end{array}$ & $\begin{array}{l}\text { Draw } \\
\text { Down } \\
\text { (m) }\end{array}$ & $\begin{array}{l}\text { V } \\
\text { Notch } \\
\text { Head } \\
(\mathrm{cm})\end{array}$ & $\begin{array}{l}\text { Debit } \\
\text { Q }\end{array}$ \\
\hline \multicolumn{7}{|c|}{$19 / 11 / 12$} \\
\hline & \multirow[t]{23}{*}{ 08:00:00 } & 0 & 16.60 & 0.00 & 12.02 & 7.23 \\
\hline & & 1 & 17.19 & 0.59 & & \\
\hline & & 2 & 17.5 & 0.90 & & \\
\hline & & 3 & 17.4 & 0.80 & & \\
\hline & & 4 & 17.4 & 0.80 & & \\
\hline & & 5 & 17.42 & 0.82 & & \\
\hline & & 6 & 17.45 & 0.85 & & \\
\hline & & 7 & 17.45 & 0.85 & & \\
\hline & & 8 & 17.45 & 0.85 & & \\
\hline & & 9 & 17.48 & 0.88 & & \\
\hline & & 10 & 17.48 & 0.88 & & \\
\hline & & 12 & 17.53 & 0.93 & & \\
\hline & & 14 & 17.53 & 0.93 & & \\
\hline & & 16 & 17.53 & 0.93 & & \\
\hline & & 18 & 17.53 & 0.93 & & \\
\hline & & 20 & 17.55 & 0.95 & & \\
\hline & & 25 & 17.6 & 1.00 & & \\
\hline & & 30 & 17.6 & 1.00 & & \\
\hline & & 35 & 17.6 & 1.00 & & \\
\hline & & 40 & 17.62 & 1.02 & & \\
\hline & & 45 & 17.65 & 1.05 & & \\
\hline & & 50 & 17.63 & 1.03 & & \\
\hline & & 55 & 17.65 & 1.05 & & \\
\hline & \multirow[t]{6}{*}{ 09:00:00 } & 60 & 17.67 & 1.07 & & \\
\hline & & 70 & 17.69 & 1.09 & & \\
\hline & & 80 & 17.69 & 1.09 & & \\
\hline & & 90 & 17.72 & 1.12 & & \\
\hline & & 100 & 17.73 & 1.13 & & \\
\hline & & 110 & 17.74 & 1.14 & & \\
\hline & \multirow[t]{3}{*}{ 10:00:00 } & 120 & 17.75 & 1.15 & & \\
\hline & & 135 & 17.8 & 1.20 & & \\
\hline & & 150 & 17.77 & 1.17 & & \\
\hline & 11:00:00 & 180 & 17.8 & 1.20 & & \\
\hline
\end{tabular}

Pumping Test Data

Step 2

\begin{tabular}{|c|c|c|c|c|c|c|}
\hline Date & Time & Min & $\begin{array}{l}\text { DWL } \\
(\mathrm{m})\end{array}$ & $\begin{array}{l}\text { Draw } \\
\text { Down } \\
\text { (m) }\end{array}$ & $\begin{array}{l}V \\
\text { Notch } \\
\text { Head } \\
(\mathrm{cm})\end{array}$ & $\begin{array}{l}\text { Deb } \\
\text { it Q } \\
\end{array}$ \\
\hline \multirow[t]{32}{*}{$19 / 11 / 12$} & $11: 00: 00$ & 0 & 17.8 & 1.2 & 14.06 & 32 \\
\hline & & 1 & 18.07 & 1.47 & & \\
\hline & & 2 & 18.07 & 1.47 & & \\
\hline & & 3 & 18.13 & 1.53 & & \\
\hline & & 5 & 18.13 & 1.53 & & \\
\hline & & 6 & 18.13 & 1.53 & & \\
\hline & & 7 & 18.13 & 1.53 & & \\
\hline & & 8 & 18.16 & 1.56 & & \\
\hline & & 9 & 18.15 & 1.55 & & \\
\hline & & 1 & 18.13 & 1.53 & & \\
\hline & & 1 & 18.19 & 1.59 & & \\
\hline & & 1 & 18.19 & 1.59 & & \\
\hline & & 1 & 18.19 & 1.59 & & \\
\hline & & 1 & 18.2 & 1.6 & & \\
\hline & & 2 & 18.18 & 1.58 & & \\
\hline & & 2 & 18.18 & 1.58 & & \\
\hline & & 3 & 18.2 & 1.6 & & \\
\hline & & 3 & 18.2 & 1.6 & & \\
\hline & & 4 & 18.2 & 1.6 & & \\
\hline & & 4 & 18.22 & 1.62 & & \\
\hline & & 5 & 18.22 & 1.62 & & \\
\hline & & 5 & 18.23 & 1.63 & & \\
\hline & 12:00:00 & 6 & 18.25 & 1.65 & & \\
\hline & & 7 & 18.25 & 1.65 & & \\
\hline & & 8 & 18.26 & 1.66 & & \\
\hline & & 9 & 18.27 & 1.67 & & \\
\hline & & 1 & 18.27 & 1.67 & & \\
\hline & & 1 & 18.29 & 1.69 & & \\
\hline & 13:00:00 & 1 & 18.27 & 1.67 & & \\
\hline & & 1 & 18.32 & 1.72 & & \\
\hline & & 1 & 18.31 & 1.71 & & \\
\hline & $14: 00: 00$ & 1 & 18.31 & 1.71 & & \\
\hline
\end{tabular}

Pumping Test Data

Step 3

\begin{tabular}{|c|c|c|c|c|c|c|}
\hline Date & Time & $\begin{array}{l}\text { Minut } \\
\mathrm{e}\end{array}$ & $\begin{array}{l}\text { DWL } \\
(\mathrm{m})\end{array}$ & $\begin{array}{l}\text { Draw } \\
\text { Down } \\
\text { (m) }\end{array}$ & $\begin{array}{l}V \\
\text { Notch } \\
\text { Head } \\
(\mathrm{cm}) \\
\end{array}$ & $\begin{array}{l}\text { Debit } \\
\text { Q } \\
\end{array}$ \\
\hline \multirow[t]{33}{*}{$19 / 11 / 12$} & $14: 00: 00$ & 0.00 & 18.31 & 1.71 & 15 & 12.11 \\
\hline & & 1.00 & 18.49 & 1.89 & & \\
\hline & & 2.00 & 18.50 & 1.90 & & \\
\hline & & 3.00 & 18.50 & 1.90 & & \\
\hline & & 4.00 & 18.50 & 1.90 & & \\
\hline & & 5.00 & 18.50 & 1.90 & & \\
\hline & & 6.00 & 18.58 & 1.98 & & \\
\hline & & 7.00 & 18.56 & 1.96 & & \\
\hline & & 8.00 & 18.54 & 1.94 & & \\
\hline & & 9.00 & 18.53 & 1.93 & & \\
\hline & & 10.00 & 18.54 & 1.94 & & \\
\hline & & 12.00 & 18.54 & 1.94 & & \\
\hline & & 14.00 & 18.54 & 1.94 & & \\
\hline & & 16.00 & 18.55 & 1.95 & & \\
\hline & & 18.00 & 18.55 & 1.95 & & \\
\hline & & 20.00 & 18.56 & 1.96 & & \\
\hline & & 25.00 & 18.57 & 1.97 & & \\
\hline & & 30.00 & 18.58 & 1.98 & & \\
\hline & & 35.00 & 18.59 & 1.99 & & \\
\hline & & 40.00 & 18.60 & 2.00 & & \\
\hline & & 45.00 & 18.60 & 2.00 & & \\
\hline & & 50.00 & 18.61 & 2.01 & & \\
\hline & & 55.00 & 18.61 & 2.01 & & \\
\hline & $15: 00: 00$ & 60.00 & 18.61 & 2.01 & & \\
\hline & & 70.00 & 18.62 & 2.02 & & \\
\hline & & 80.00 & 18.64 & 2.04 & & \\
\hline & & 90.00 & 18.64 & 2.04 & & \\
\hline & & 100.00 & 18.65 & 2.05 & & \\
\hline & & 110.00 & 18.67 & 2.07 & & \\
\hline & $16: 00: 00$ & 120.00 & 18.68 & 2.08 & & \\
\hline & & 135.00 & 18.68 & 2.08 & & \\
\hline & & 150.00 & 18.72 & 2.12 & & \\
\hline & $17: 00: 00$ & 180.00 & 18.73 & 2.13 & & \\
\hline
\end{tabular}

Pumping Test Data

Step 4

\begin{tabular}{|c|c|c|c|c|c|c|}
\hline Date & Time & Minute & $\begin{array}{l}\text { DWL } \\
\text { (m) }\end{array}$ & $\begin{array}{l}\text { Draw } \\
\text { Down } \\
\text { (m) }\end{array}$ & $\begin{array}{l}V \\
\text { Notch } \\
\text { Head } \\
\mathrm{Icm} \\
\end{array}$ & $\begin{array}{l}\text { Debit } \\
\text { Q }\end{array}$ \\
\hline \multirow[t]{33}{*}{$19 / 11 / 12$} & $17: 00: 00$ & 0 & 19.05 & 2.45 & 15.5 & 13.15 \\
\hline & & 1 & 19.05 & 2.45 & & \\
\hline & & 2 & 19.07 & 2.47 & & \\
\hline & & 3 & 19.07 & 2.47 & & \\
\hline & & 4 & 19.07 & 2.47 & & \\
\hline & & 5 & 19.08 & 2.48 & & \\
\hline & & 6 & 19.08 & 2.48 & & \\
\hline & & 7 & 19.07 & 2.47 & & \\
\hline & & 8 & 19.07 & 2.47 & & \\
\hline & & 9 & 19.08 & 2.48 & & \\
\hline & & 10 & 19.09 & 2.49 & & \\
\hline & & 12 & 19.09 & 2.49 & & \\
\hline & & 14 & 19.1 & 2.50 & & \\
\hline & & 16 & 19.1 & 2.50 & & \\
\hline & & 18 & 19.11 & 2.51 & & \\
\hline & & 20 & 19.11 & 2.51 & & \\
\hline & & 25 & 19.12 & 2.52 & & \\
\hline & & 30 & 19.14 & 2.54 & & \\
\hline & & 35 & 19.14 & 2.54 & & \\
\hline & & 40 & 19.18 & 2.58 & & \\
\hline & & 45 & 19.2 & 2.60 & & \\
\hline & & 50 & 19.2 & 2.60 & & \\
\hline & & 55 & 19.21 & 2.61 & & \\
\hline & $18: 00: 00$ & 60 & 19.22 & 2.62 & & \\
\hline & & 70 & 19.3 & 2.70 & & \\
\hline & & 80 & 19.34 & 2.74 & & \\
\hline & & 90 & 19.36 & 2.76 & & \\
\hline & & 100 & 19.38 & 2.78 & & \\
\hline & & 110 & 19.41 & 2.81 & & \\
\hline & 19:00:00 & 120 & 19.42 & 2.82 & & \\
\hline & & 135 & 19.45 & 2.85 & & \\
\hline & & 150 & 19.46 & 2.86 & & \\
\hline & $20: 00: 00$ & 180 & 19.5 & 2.90 & & \\
\hline
\end{tabular}


Appendix 2. Recovery Test of KKT DWW

\# 01

\begin{tabular}{|c|c|c|c|c|c|c|}
\hline Date & Time & $\begin{array}{l}\text { Minute } \\
\text { (t) }\end{array}$ & :' & $t / t^{\prime}$ & $\begin{array}{l}\text { DWL } \\
(\mathrm{m})\end{array}$ & $\begin{array}{l}\text { Residual } \\
\text { Draw } \\
\text { Down } \\
\text { (m) } \\
\end{array}$ \\
\hline \multirow[t]{35}{*}{$19 / 11 / 12$} & 20:00:00 & 720 & 0 & 0.00 & 19.50 & 2.90 \\
\hline & & 721 & 1 & 721.00 & 18.50 & 1.90 \\
\hline & & 722 & 2 & 361.00 & 18.20 & 1.60 \\
\hline & & 723 & 3 & 241.00 & 18.06 & 1.46 \\
\hline & & 724 & 4 & 181.00 & 17.96 & 1.36 \\
\hline & & 725 & 5 & 145.00 & 17.91 & 1.31 \\
\hline & & 726 & 6 & 121.00 & 17.86 & 1.26 \\
\hline & & 727 & 7 & 103.86 & 17.82 & 1.22 \\
\hline & & 728 & 8 & 91.00 & 17.78 & 1.18 \\
\hline & & 729 & 9 & 81.00 & 17.77 & 1.17 \\
\hline & & 730 & 10 & 73.00 & 17.75 & 1.15 \\
\hline & & 732 & 12 & 61.00 & 17.71 & 1.11 \\
\hline & & 734 & 14 & 52.43 & 17.68 & 1.08 \\
\hline & & 736 & 16 & 46.00 & 17.65 & 1.05 \\
\hline & & 738 & 18 & 41.00 & 17.63 & 1.03 \\
\hline & & 740 & 20 & 37.00 & 17.61 & 1.01 \\
\hline & & 745 & 25 & 29.80 & 17.52 & 0.92 \\
\hline & & 750 & 30 & 25.00 & 17.50 & 0.90 \\
\hline & & 755 & 35 & 21.57 & 17.46 & 0.86 \\
\hline & & 760 & 40 & 19.00 & 17.43 & 0.83 \\
\hline & & 765 & 45 & 17.00 & 17.41 & 0.81 \\
\hline & & 770 & 50 & 15.40 & 17.39 & 0.79 \\
\hline & & 775 & 55 & 14.09 & 17.36 & 0.76 \\
\hline & 21:00:00 & 780 & 60 & 13.00 & 17.34 & 0.74 \\
\hline & & 790 & 70 & 11.29 & 17.28 & 0.68 \\
\hline & & 800 & 80 & 10.00 & 17.24 & 0.64 \\
\hline & & 810 & 90 & 9.00 & 17.20 & 0.60 \\
\hline & & 820 & 100 & 8.20 & 17.17 & 0.57 \\
\hline & & 830 & 110 & 7.55 & 17.13 & 0.53 \\
\hline & 22:00:00 & 840 & 120 & 7.00 & 17.09 & 0.49 \\
\hline & & 855 & 135 & 6.33 & 17.05 & 0.45 \\
\hline & & 870 & 150 & 5.80 & 17.01 & 0.41 \\
\hline & & 885 & 165 & 5.36 & 16.99 & 0.39 \\
\hline & 23:00:00 & 900 & 180 & 5.00 & 16.96 & 0.36 \\
\hline & & 930 & 210 & 4.43 & 16.93 & 0.33 \\
\hline \multirow[t]{41}{*}{ 20/11/12 } & 24:00:00 & 960 & 240 & 4.00 & 16.87 & 0.27 \\
\hline & & 990 & 270 & 3.67 & 16.80 & 0.20 \\
\hline & 01:00:00 & 1020 & 300 & 3.40 & 16.75 & 0.15 \\
\hline & & 1050 & 330 & 3.18 & 16.70 & 0.10 \\
\hline & 02:00:00 & 1080 & 360 & 3.00 & 16.62 & 0.02 \\
\hline & & 1110 & 390 & 2.85 & 16.61 & 0.01 \\
\hline & 03:00:00 & 1140 & 420 & 2.71 & 16.55 & -0.05 \\
\hline & & 1170 & 450 & 2.60 & 16.55 & -0.05 \\
\hline & 04:00:00 & 1200 & 480 & 2.50 & 16.55 & -0.05 \\
\hline & & 1230 & 510 & 2.41 & 16.50 & -0.10 \\
\hline & 05:00:00 & 1260 & 540 & 2.33 & 16.50 & -0.10 \\
\hline & & 1290 & 570 & 2.26 & 16.50 & -0.10 \\
\hline & 06:00:00 & 1320 & 600 & 2.20 & 16.45 & -0.15 \\
\hline & & 1350 & 630 & 2.14 & 16.45 & -0.15 \\
\hline & 07:00:00 & 1380 & 660 & 2.09 & 16.45 & -0.15 \\
\hline & & 1410 & 690 & 2.04 & 16.45 & -0.15 \\
\hline & 08:00:00 & 1440 & 720 & 2.00 & 16.45 & -0.15 \\
\hline & & 1470 & 750 & 1.96 & 16.40 & -0.20 \\
\hline & 09:00:00 & 1500 & 780 & 1.92 & 16.40 & -0.20 \\
\hline & & 1530 & 810 & 1.89 & 16.40 & -0.20 \\
\hline & 10:00:00 & 1560 & 840 & 1.86 & 16.40 & -0.20 \\
\hline & & 1590 & 870 & 1.83 & 16.35 & -0.25 \\
\hline & 11:00:00 & 1620 & 900 & 1.80 & 16.35 & -0.25 \\
\hline & & 1650 & 930 & 1.77 & 16.35 & -0.25 \\
\hline & $12: 00: 00$ & 1680 & 960 & 1.75 & 16.35 & -0.25 \\
\hline & & 1710 & 990 & 1.73 & 16.35 & -0.25 \\
\hline & 13:00:00 & 1740 & 1020 & 1.71 & 16.30 & -0.30 \\
\hline & & 1770 & 1050 & 1.69 & 16.30 & -0.30 \\
\hline & 14:00:00 & 1800 & 1080 & 1.67 & 16.30 & -0.30 \\
\hline & & 1830 & 1110 & 1.65 & 16.30 & -0.30 \\
\hline & $15: 00: 00$ & 1860 & 1140 & 1.63 & 16.25 & -0.35 \\
\hline & & 1890 & 1170 & 1.62 & 16.25 & -0.35 \\
\hline & $16: 00: 00$ & 1920 & 1200 & 1.60 & 16.25 & -0.35 \\
\hline & & 1950 & 1230 & 1.59 & 16.20 & -0.40 \\
\hline & 17:00:00 & 1980 & 1260 & 1.57 & 16.20 & -0.40 \\
\hline & & 2010 & 1290 & 1.56 & 16.20 & -0.40 \\
\hline & 18:00:00 & 2040 & 1320 & 1.55 & 16.15 & -0.45 \\
\hline & & 2070 & 1350 & 1.53 & 16.15 & -0.45 \\
\hline & 19:00:00 & 2100 & 1380 & 1.52 & 16.15 & -0.45 \\
\hline & & 2130 & 1410 & 1.51 & 16.10 & -0.50 \\
\hline & 20:00:00 & 2160 & 1440 & 1.50 & 16.10 & -0.50 \\
\hline
\end{tabular}

Note $\mathrm{t}=$ time after pump is switched on

$\mathrm{t}^{\prime}=$ time after pump is switched off 